\title{
Highly sensitive terahertz measurement of layer thickness using a two-cylinder waveguide sensor
}

\author{
M. Theuer, ${ }^{1,2}$ R. Beigang, ${ }^{2,3}$ and D. Grischkowsky ${ }^{1, a)}$ \\ ${ }^{1}$ School of Electrical and Computer Engineering, Oklahoma State University, Stillwater, \\ Oklahoma 74078, USA \\ ${ }^{2}$ Department of Terahertz Measurement and Systems, Fraunhofer Institute for Physical Measurement \\ Techniques, Kaiserslautern, Rhineland-Palatinate 67663, Germany \\ ${ }^{3}$ Department of Physics, University of Kaiserslautern, Kaiserslautern, Rhineland-Palatinate 67663, \\ Germany
}

(Received 23 June 2010; accepted 30 July 2010; published online 17 August 2010)

\begin{abstract}
We report on the layer thickness determination on dielectrically coated metal cylinders using terahertz $(\mathrm{THz})$ time-domain spectroscopy. A considerable sensitivity increase of up to a factor of 150 is obtained for layers down to $2.5 \mu \mathrm{m}$ thickness by introducing an experimental geometry based on a two-cylinder waveguide sensor. The layer attached on one metal cylinder is guided in contact with the second metal cylinder in the $\mathrm{THz}$ beam waist. This approach uses concepts of adiabatic $\mathrm{THz}$ wave compression and the advantages of $\mathrm{THz}$ waveguides. The results are compared to measurements on free-standing layers. (c) 2010 American Institute of Physics.
\end{abstract}

[doi:10.1063/1.3481080]

Thin film sensing and layer thickness determination are important for various industrial processes. The terahertz $(\mathrm{THz})$ frequency band (located between $100 \mathrm{GHz}$ and 10 $\mathrm{THz}$, corresponding to wavelengths between 3000 and $30 \mu \mathrm{m}$ in free-space) efficiently penetrates most dielectrics. Thus, $\mathrm{THz}$ nondestructive testing can be used to determine the thickness of common plastics ${ }^{1}$ and paint layers. ${ }^{2}$ Various techniques have been reported to improve the detection threshold for $\mathrm{THz}$ thin film sensing, e. g., evaluation of difference signals, ${ }^{3}$ resonance changes of metamaterials, ${ }^{4}$ or the application of metal meshes to enhance the amplitude reduction of the $\mathrm{THz}$ bandpass. ${ }^{5}$

The determination of the thickness is typically carried out by measuring the $\mathrm{THz}$ pulse delay of a sample. ${ }^{6}$ This can be done using $\mathrm{THz}$ time-domain spectroscopy (THz-TDS) systems based on femtosecond lasers. ${ }^{7}$ The electric field is measured by sampling the $\mathrm{THz}$ pulse as a function of delay, typically using a mechanical delay line. Thus, the thickness, $\mathrm{T}$, of an introduced sample [see experimental setup Fig. 1(a)] can be derived in a transmission measurement by

$$
\mathrm{T}=\mathrm{c} \Delta \mathrm{t} /(\mathrm{n}-1),
$$

where $\mathrm{n}$ is the refractive index of the sample, $\Delta \mathrm{t}$ the measured pulse delay and $\mathrm{c}$ the velocity of light.

Waveguide geometries based on slowly varying metal surfaces have recently attracted interest in the THz range. ${ }^{8-13}$ Using these results, we propose and demonstrate improved thin film sensing of plastic coatings.

The main problem to solve is the measurement of a delay change much below the applied pulse duration of 1 ps. The high signal-to-noise ratio of the $\mathrm{THz}$ system allows for the accurate determination of the pulse delay at the peak maximum. By evaluation of the steep edges of the pulse at the zero crossings of the electric field, the observational accuracy can be even more increased. Besides random errors, which can be reduced by averaging, systematic errors con-

${ }^{a}$ Electronic mail: daniel.grischkowsky@okstate.edu. tribute to the uncertainty of the measured quantities. Delay lines, for instance, have mechanical specifications with an accuracy between 0.1 and $1 \mu \mathrm{m}$. However, the largest influence is due to thermal drifts, ${ }^{14}$ which requires frequent reference scans to minimize the accumulated error. ${ }^{15}$ The reference zero delay of our system drifts by approximately 20 $\mathrm{fs} / \mathrm{h}$. This is reasonable, as the free-space optics is mounted on a stainless-steel table (thermal expansion coefficient $\left.17.3 \mu \mathrm{m} / \mathrm{m}{ }^{\circ} \mathrm{C}\right)$. With a $1 \mathrm{~m}$ propagation distance between optics this delay drift corresponds to a thermal drift of $0.3^{\circ} \mathrm{C}$, which is a typical temperature oscillation of an airconditioned laboratory. This means that under optimal con-

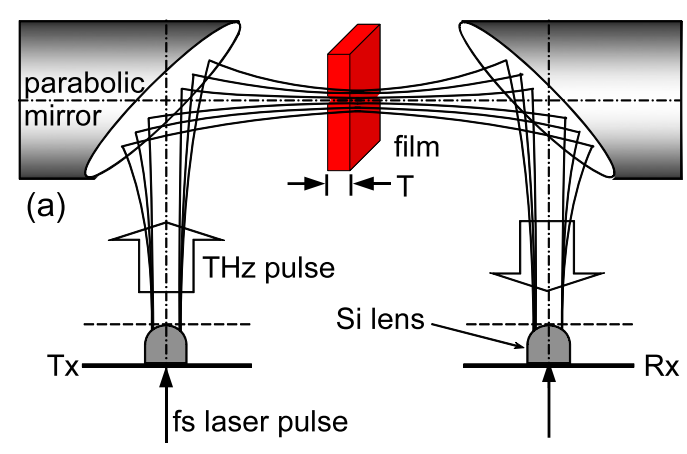

(b)

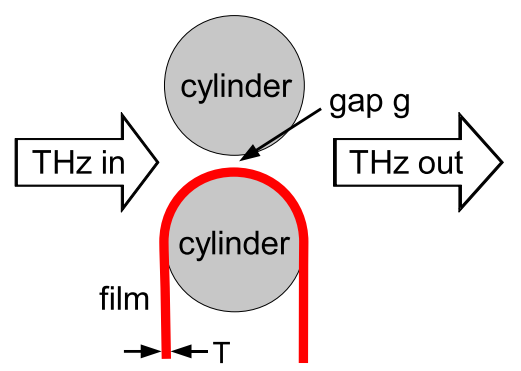

FIG. 1. (Color online) (a) THz-TDS setup with the beam waist centered between the two parabolic mirrors. A film sample is placed in a single-pass transmission geometry. (b) Layout of the two-cylinder waveguide sensor. The $\mathrm{THz}$ wave passes through the subwavelength gap, g, where an intense interaction with the film occurs. 


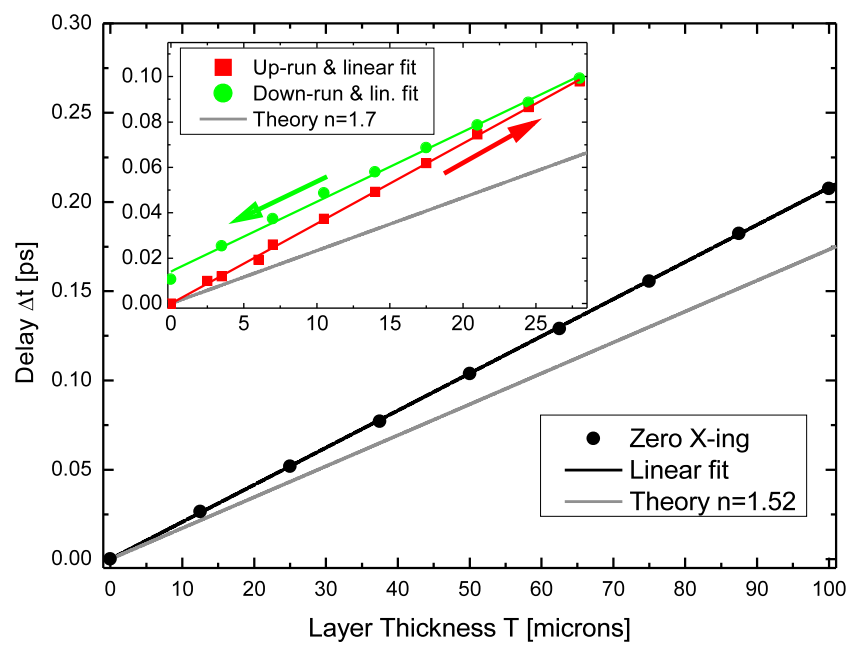

FIG. 2. (Color online) Pulse delay added by introducing PE layers $\left(\mathrm{n}_{\mathrm{PE}}\right.$ $=1.52,12.5 \mu \mathrm{m}$ thick) into the THz beam, as shown in Fig. 1(a). The theoretical curve and the linear fit show a slightly different inclination. Within the measurement accuracy of the geometrical thickness a good agreement with the theoretical values is obtained. Inset: Same measurement for thin PET layers $\left[\mathrm{n}_{\mathrm{PET}}=1.7,2.5(3.5) \mu \mathrm{m}\right.$ thick$]$.

ditions, long-term stability limits a reliable investigation of sub-10 $\mu \mathrm{m}$ thin film samples within one measurement series. Therefore, it is desirable to increase the measured delay per micron of sample to enhance the sensitivity of THz-TDS for thin film sensing to minimize the negative influence of thermal drifts, especially if stable measurements are necessary.

Starting with the standard approach of measuring the single-pass delay, two types of layers were investigated. The thinnest available layers were Mylar ${ }^{\circledR}$ films with thicknesses of 2.5 or $3.5 \mu \mathrm{m}$ and consisting of polyethylene terephthalate (PET) $\left[\mathrm{n}_{\mathrm{PET}}=1.7\right.$ (Ref. 16)]. For comparison, a thicker layer polyethylene (PE) cling wrap with $12.5 \mu \mathrm{m}$ thickness was also used $\left[\mathrm{n}_{\mathrm{PE}}=1.52\right.$ (Ref. 16)]. Stacks of these dielectric films were measured with high temporal resolution.

The results of the free-standing samples are shown in Fig. 2. The large plot shows the measured single-pass delay for the PE cling wrap and the inset for the PET Mylar ${ }^{\circledR}$, respectively. The theoretical curves, according to Eq. (1) with $\mathrm{n}_{\mathrm{PE}}=1.52$ and $\mathrm{n}_{\mathrm{PET}}=1.7$, are plotted for comparison along with the linear fit of the measured data points. Within the experimental accuracy, the measured delay and the theoretical values correspond rather well for thick layers. A linear fit of the precise delay values for the zero crossings (black dots) indicates that this type of measurement can be precise for layers above $10 \mu \mathrm{m}$. The different pulse-delay factors of $2.1 \mathrm{fs} / \mu \mathrm{m}$ for the measurement and $1.7 \mathrm{fs} / \mu \mathrm{m}$ for theory indicate that the film was $15.1 \mu \mathrm{m}$ thick, instead of the expected $12.5 \mu \mathrm{m}$. This discrepancy is within the manufacturing specifications (0.5 mil, approximately $12.5 \mu \mathrm{m})$ for this commercial cling wrap.

The systematic error increases considerably for layers below $10 \mu \mathrm{m}$ as seen in the inset. The measurement series of increasing (up-run; red squares) and decreasing (downrun; green dots) layer thicknesses were recorded during one continuous measurement. Although both series show a linear behavior of delay versus layer thickness, the deviation from theory is rather large. The theoretical pulse-delay factor is $2.3 \mathrm{fs} / \mu \mathrm{m}$, while for the measured up-run the factor is 3.5 and $3.1 \mathrm{fs} / \mu \mathrm{m}$ for the down-run. The reason for the increasing delay is not entirely due to extra sample thickness; the thermal drift of the reference zero of the delay was quite significant during the measurement time of approximately three hours. This is shown by the fact that the reverse downrun to lower thicknesses does not end with an identical 0 ps reference for an empty reference chamber. The total reference drift for the series according to the linear fit for decreasing layer thicknesses was 0.014 ps corresponding to a layer thickness of $6.0 \mu \mathrm{m}$. So for the down-run measured $2.5 \mu \mathrm{m}$ layer sample, this gives a relative error above $200 \%$. However, it is possible to subtract the linear (with measurement time) thermal drift from the data points to achieve consistency and then the up-run and down-run data will overlap.

The simple two-cylinder waveguide sensor demonstrated in this paper brings a considerable increase in sensitivity. This approach combines our previous work on $\mathrm{THz}$ wave compression using metal flares ${ }^{12}$ and the sensing capabilities of THz surface waveguides. ${ }^{13}$ The experimental layout, schematically shown in Fig. 1(b), operates as an exchangeable sensor for a standard THz-TDS system. The film under investigation is wrapped on the surface of one metal cylinder. Together with the opposing metal cylinder (both $63 \mathrm{~mm} \mathrm{di-}$ ameter), the $\mathrm{THz}$ optics are formed with the coated and the uncoated cylinders mounted in direct contact or with a preset gap defined by spacers. The whole assembly is placed into the center beam waist of the THz-TDS system, replacing the film sample shown in Fig. 1(a).

Using the two-cylinder waveguide sensor, the approaching $\mathrm{THz}$ wave is spatially compressed from a wavelength dependent spot-size $(9 \mathrm{~mm}$ diameter at $1 \mathrm{THz})$ to a subwavelength line focus (less than $10 \mu \mathrm{m}$ ). After the $\mathrm{THz}$ wave propagates through the dielectrically filled gap the metal surfaces of the two facing cylinders continue to act like a horn, as the expanding wave is guided out of the sensor. The high efficiency coupling is due to the beam pattern changing adiabatically, i. e., slowly varying with respect to the wavelength. ${ }^{8}$

During the propagation through the gap between the cylinders the interaction between the $\mathrm{THz}$ pulse and the coating is very intense, since this scheme is comparable to a parallelplate waveguide. ${ }^{17,18}$ In this arrangement the $\mathrm{THz}$ wave has a much longer propagation length within the film compared to the single-pass case, thereby giving rise to a considerably increased delay. This scheme transforms the problem from a single-pass perpendicular transmission into an in-plane propagation. The geometry of commercial metal cylinders is reproducible, easy to align, and does not require additional optical components.

Even if the measurement is carried out in mechanical contact $(\mathrm{g}=0 \mu \mathrm{m})$ between the two polished cylinders, due to the machining accuracy of the cylinders (axis, tilt of flat end surfaces), the residual small angle between the two cylinder axes produces a tiny gap. Additional contact pressure and independent mounts decrease this gap considerably. In Fig. 3, the results measured with the two-cylinder sensor are compared to the theory curves. Without air gap $(\mathrm{g}=0 \mu \mathrm{m})$, theory predicts larger delays than observed, especially for thin layers. This discrepancy is caused by the surface roughness of the cylinders. Although the soft flexible plastic foil can further reduce the gap, due to the limited foil thickness, there are still small air spaces remaining $\Delta$ n averaged tiny 


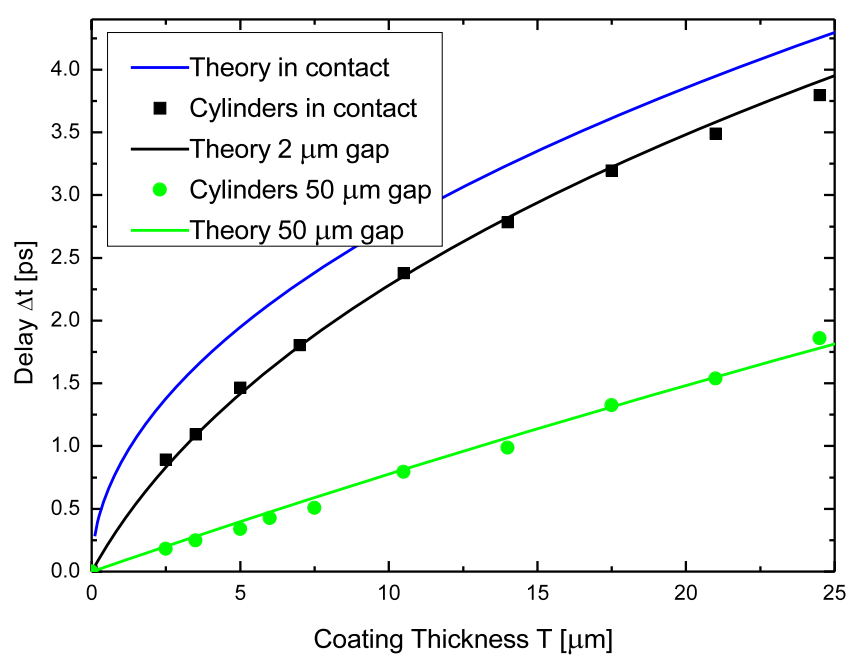

FIG. 3. (Color online) Pulse delay caused by the two-cylinder waveguide sensor replacing the film sample of Fig. 1(a) in the THz beam. On one of the $63 \mathrm{~mm}$ diameter metal cylinders stepwise 2.5(3.5) $\mu \mathrm{m}$ thick PET layers are attached. One series was recorded with the cylinders in contact (black dots) and another one with a $50 \mu \mathrm{m}$ gap (green circles). Theoretical curves are shown for comparison. The pulse delay for the $2.5 \mu \mathrm{m}$ layer on the sample cylinder is increased by a factor of 150 compared to the theoretical singlepass delay value, shown in Fig. 2.

gap $(\mathrm{g}=2 \mu \mathrm{m})$ in the order of the surface roughness shows good agreement with the measured values.

A second series was recorded with spacers between the cylinders forming a $50 \mu \mathrm{m}$ gap. These gaps are used to calculate the theoretical values of the delay, assuming a simple model using formulas known for partially filled parallel-plate waveguides. The delay is integrated from the initial compression through the intense interaction in the vicinity of the gap and to the exit expansion. ${ }^{13}$

For the in-contact pressed cylinders and the corresponding theory curve $(\mathrm{g}=2 \mu \mathrm{m})$, the values match for thin layers, while for thick layers a transition into saturation can be observed. Working with a larger gap $(\mathrm{g}=50 \mu \mathrm{m})$, theory predicts a nearly linear behavior of the delay as a function of coating thickness, which is also in good agreement with the measured values. This type of measurement can be used to obtain a simple calibration curve and good sensitivity at the same time. The sensitivity increase given by the ratio of measured stretched delay of the two-cylinder waveguide sensor to the theoretical single-pass delay reaches up to 150 for the $2.5 \mu \mathrm{m}$ coating and the cylinders in contact, dropping to 66 for a $24.5 \mu \mathrm{m}$ coating. In the case of a $50 \mu \mathrm{m}$ gap, the pulse-delay factor is $74.1 \mathrm{fs} / \mu \mathrm{m}$ which corresponds to a sensitivity increase by a factor of 32 .

Also, the measurement of thin coatings on metal foils is now possible, even if the perpendicular reflection cannot resolve the two originating echo pulses. These measurements would be performed by attaching the foil to the sample cylinder facing the coated side toward the other cylinder.

As shown above, even a small gap reduces the delay of the $\mathrm{THz}$ pulse by reducing the filling factor, especially in the strong interaction area of the gap. This fact requires good mechanical accuracy for the cylinders and their mounts. The cylinders used in our experiment were machined with standard production accuracy. Although the inherent gap reduces the filling factor of the waveguide and thereby decreases the delay, it is still acceptable, shows a much higher delay than the single-pass, and remains constant during the measurements. Consequently, a calibration curve can determine the pulse delay per micron for a particular setup. For applications, a calibration curve for the actual gap and sample has to be recorded to determine the sample thickness. In our experiments the positioning accuracy of the cylinders in the beam waist was not very critical as shown by a reproducibility check and the linear behavior. The limit of this technique for the detection of thin layers is no longer given by the longterm thermal stability of the $\mathrm{THz}$ system but is defined by the accuracy of the gap and the sample mount. Therefore, we believe that detection of layers below $1 \mu \mathrm{m}$ is feasible with the two-cylinder waveguide sensor as demonstrated by our ability to measure delays of less than 0.1 ps shown in Fig. 2 .

In conclusion, we have introduced a two-cylinder waveguide sensor which extends the scope of $\mathrm{THz}$ thin film sensing into the submicron range. A 150 times higher sensitivity than in the standard approach of single-pass measurement has been obtained. The scheme can be easily adapted to industrial needs as an independent sensor (e. g., attached to a production line where a coated foil is guided around deflection rolls). No additional optical components are necessary, since the metal cylinders guide the $\mathrm{THz}$ beam.

This work was partially supported by the Fraunhofer Gesellschaft FhG internal program for the scientific exchange (PROF. $\mathrm{X}^{2}$ ) and the National Science Foundation.

${ }^{1}$ D. Mittleman, S. Hunsche, L. Boivin, and M. Nuss, Opt. Lett. 22, 904 (1997).

${ }^{2}$ T. Yasui, T. Yasuda, K. Sawanaka, and T. Araki, Appl. Opt. 44, 6849 (2005).

${ }^{3}$ Z. Jiang, M. Li, and X.-C. Zhang, Appl. Phys. Lett. 76, 3221 (2000).

${ }^{4}$ J. F. O’Hara, R. Singh, I. Brener, E. Smirnova, J. Han, A. J. Taylor, and W. Zhang, Opt. Express 16, 1786 (2008).

${ }^{5}$ S. Yoshida, E. Kato, K. Suizu, Y. Nakagomi, Y. Ogawa, and K. Kawase, Appl. Phys. Express 2, 012301 (2009).

${ }^{6}$ B. Pradarutti, R. Müller, G. Matthäus, C. Brückner, S. Riehemann, G. Notni, S. Nolte, and A. Tünnermann, Opt. Express 15, 17652 (2007).

${ }^{7}$ D. Grischkowsky, S. Keiding, M. Exter, and C. Fattinger, J. Opt. Soc. Am. B 7, 2006 (1990)

${ }^{8}$ J. Zhang and D. Grischkowsky, Appl. Phys. Lett. 86, 061109 (2005).

${ }^{9}$ S.-H. Kim, E. S. Lee, Y. B. Ji, and T.-I. Jeon, Opt. Express 18, 1289 (2010).

${ }^{10}$ H. Zhan, R. Mendis, and D. M. Mittleman, Opt. Express 18, 9643 (2010).

${ }^{11}$ Y. Avetisyan, H. Hakopyan, A. Makaryan, and K. Nerkararyan, Armenian J. Phys. 3, 69 (2010).

${ }^{12}$ M. Theuer, R. Beigang, and D. Grischkowsky, Appl. Phys. Lett. 96, 191110 (2010).

${ }^{13}$ M. Theuer, R. Beigang, and D. Grischkowsky, Opt. Express 18, 11456 (2010).

${ }^{14}$ W. Withayachumnankul, B. M. Fischer, H. Lin, and D. Abbott, J. Opt. Soc. Am. B 25, 1059 (2008).

${ }^{15}$ S. R. Tripathi, M. Aoki, K. Mochizuki, I. Hosako, T. Asahi, and N. Hiromoto, Opt. Commun. 283, 2488 (2010).

${ }^{16}$ D. Smith and E. Loewenstein, Appl. Opt. 14, 1335 (1975).

${ }^{17}$ R. Mendis and D. Grischkowsky, Opt. Lett. 26, 846 (2001).

${ }^{18}$ J. Zhang and D. Grischkowsky, Opt. Lett. 29, 1617 (2004). 